\title{
LAZY Gene Family in Plant Gravitropism
}

\author{
Zhicheng Jiao ${ }^{1,2}$, Huan Du ${ }^{1,2}$, Shu Chen ${ }^{1,2}$, Wei Huang ${ }^{3,4}$ and Liangfa Ge $e^{1,2,5 *}$ \\ ${ }^{1}$ College of Forestry and Landscape Architecture, South China Agricultural University, Guangzhou, China, ${ }^{2}$ Guangdong Key \\ Laboratory for Innovative Development and Utilization of Forest Plant Germplasm, South China Agricultural University, \\ Guangzhou, China, ${ }^{3}$ State Key Laboratory for Conservation and Utilization of Subtropical Agro-bioresources, College of Life \\ Sciences, South China Agricultural University, Guangzhou, China, ${ }^{4}$ Guangdong Provincial Key Laboratory of Protein \\ Function and Regulation in Agricultural Organisms, College of Life Sciences, South China Agricultural University, Guangzhou, \\ China, ${ }^{5}$ The Guangdong Subcenter of the National Center for Soybean Improvement, College of Agriculture, South China \\ Agricultural University, Guangzhou, China
}

OPEN ACCESS

Edited by:

Patrick H. Masson

University of Wisconsin-Madison,

United States

Reviewed by:

Jie Le,

Key Laboratory of Plant Molecular Physiology, Institute of Botany (CAS),

China

Jozef Mravec,

University of Copenhagen, Denmark

${ }^{*}$ Correspondence: Liangfa Ge

lge@scau.edu.cn

Specialty section: This article was submitted to Plant Physiology, a section of the journal

Frontiers in Plant Science

Received: 14 September 2020

Accepted: 01 December 2020

Published: 15 January 2021

Citation:

Jiao Z, Du H, Chen S, Huang W and Ge L (2021) LAZY Gene Family

in Plant Gravitropism.

Front. Plant Sci. 11:606241.

doi: 10.3389/fpls.2020.606241
Adapting to the omnipresent gravitational field was a fundamental basis driving the flourishing of terrestrial plants on the Earth. Plants have evolved a remarkable capability that not only allows them to live and develop within the Earth's gravity field, but it also enables them to use the gravity vector to guide the growth of roots and shoots, in a process known as gravitropism. Triggered by gravistimulation, plant gravitropism is a highly complex, multistep process that requires many organelles and players to function in an intricate coordinated way. Although this process has been studied for several 100 years, much remains unclear, particularly the early events that trigger the relocation of the auxin efflux carrier PIN-FORMED (PIN) proteins, which presumably leads to the asymmetrical redistribution of auxin. In the past decade, the LAZY gene family has been identified as a crucial player that ensures the proper redistribution of auxin and a normal tropic response for both roots and shoots upon gravistimulation. LAZY proteins appear to be participating in the early steps of gravity signaling, as the mutation of $L A Z Y$ genes consistently leads to altered auxin redistribution in multiple plant species. The identification and characterization of the $L A Z Y$ gene family have significantly advanced our understanding of plant gravitropism, and opened new frontiers of investigation into the novel molecular details of the early events of gravitropism. Here we review current knowledge of the LAZY gene family and the mechanism modulated by LAZY proteins for controlling both roots and shoots gravitropism. We also discuss the evolutionary significance and conservation of the LAZY gene family in plants.

Keywords: gravitropism, LAZY, auxin, PIN, gene family

\section{PLANT GRAVITROPISM}

The growth angle of plant organs is a fundamental component of plants' shoot and root system architecture, and it largely defines the space that a plant can access. The angle of a plant organ is influenced by both its intrinsic genetic nature and a variety of environmental factors, including gravitation pull, light direction, temperature, and wind. Within the gravity field, plants can sense

Abbreviations: RSA, root system architecture; GSA, gravitropic set-point angles; PAT, polar auxin transport; SOR, soilsurface roots; $\mathrm{BiFC}$, bimolecular fluorescence complementation; NLS, nuclear localization signal; GFP, green fluorescence protein; MTFs, membrane-bound transcription factors; QTL, quantitative trait locus; RNAi, RNA interference; WGD, wholegenome duplications; $5^{\prime} \mathrm{UTR}$, five prime untranslated regions. 
gravitational pull and adjust their organs' growth direction accordingly. Consequently, plant organs are developed and maintained at particular angles, which are called the gravitropic set-point angles (GSAs) (Digby and Firn, 1995; Figure 1A).

Plant gravitropism can either be a positive or a negative response (Dong et al., 2013). The positive gravitropic response occurs in the belowground root system, which re-orientates the root-tip growth angle toward the GSA and allows the roots to penetrate deep into the soil to anchor the plant and uptake nutrients and water. By contrast, the negative gravitropic response occurs in the aboveground shoot system, which grows up, against the gravity vector toward the sky to obtain space and sunlight for photosynthesis and reproduction.

Plant gravitropism comprises four successive steps: gravity perception, signal transduction that converts the gravity stimulus into physiological signals, polar auxin transport (PAT) that asymmetrically distributes auxin to the responding organs, and differential elongation of cells leading to the curvature of organs. How exactly the plant perceives the gravitational pull is not yet fully understood. The starch-statolith theory posits that gravity is perceived by the statoliths within the statocyte cells. These statocytes contain amyloplasts, which are filled with dense starch, thereby conferring to them a greater density than the surrounding cytoplasm (Baldwin et al., 2013; Su et al., 2017). Upon encountering gravitational pull, the amyloplasts can rapidly descend toward the new bottom of the cell, triggering the downstream gravitropism signal transduction via an as of yet unclear mechanism. Notably, the sedimentation process of the amyloplasts is finely tuned by the actin microfilaments network (Hou et al., 2003; Leitz et al., 2009; Blancaflor, 2013). In roots, the amyloplasts' sedimentation occurs in the columella cells. Following downward migration and settling of amyloplasts in the columella cells, this physical stimulus is transduced into a physiological and biochemical signal (Figure 1B). Unfortunately, the nature and mechanism of that signal remain unresolved. It has been proposed that the signal is capable of triggering the lateral relocation of LAZY proteins and auxin transporters (Friml et al., 2002; Furutani et al., 2020), which further asymmetrically transport auxin to the elongation zone, where the root tip bends toward the gravity vector (Blancaflor and Masson, 2003; Morita and Tasaka, 2004; Blancaflor, 2013). In shoots, negative gravitropism takes place in several tissues, including inflorescence stems, epicotyls, and hypocotyls in dicots, and the culms, mesocotyl-coleoptiles, and pulvini in monocots (Imagawa et al., 1991; Blancaflor and Masson, 2003; Morita and Tasaka, 2004; Haga and Iino, 2006; Li et al., 2007; Blancaflor, 2013; Dong et al., 2013). In these tissues, the amyloplasts' sedimentation has been directly observed in certain types of cells (such as the endodermis) (Morita and Tasaka, 2004; Saito et al., 2005; Swarup et al., 2005; Su et al., 2017). The sediment of amyloplasts in shoot tissues then triggers the lateral transport of auxin across the organ by an unclear mechanism, which leads to the asymmetrical accumulation of auxin in the opposite flanks of the responding organ, and ultimately curvature of the organ toward the GSA within hours (Su et al., 2017).

\section{GRAVITROPISM REGULATES PLANT ARCHITECTURE AND PERFORMANCE}

Gravitropism has a profound influence on both the shoot and root systems' architecture. As the most stable environmental signal, the gravity vector continuously guides the growth direction of all plant organs, thus shaping not only the overall form of a given plant but also the various characteristics of its organs, such as their growth angle, orientation, and pattern. Many studies have reported on how an altered responsiveness to the gravistimulation can lead to varied plant architectures. For example, a mutation of Loose Plant Architecture1 (LPA1) affected the perception of gravistimulation, resulting in a loose shoot architecture forming in rice, in contrast to the rather compact shoot system of the wild type (Wu et al., 2013). In roots, the strength of their gravitropic response largely defines the roottip growth angle, root depth, and distribution of roots in the soil. Disrupting or reducing gravitropism could lead to roots failing to respond properly to the perceived gravitational pull or a shallow root depth (Luschnig et al., 1998; Muller et al., 1998; Rouse et al., 1998; Kitomi et al., 2020). In nature, roots display highly varied depths (Figure 2), which convey divergent or disparate genetic abilities to perform gravitropism. Rooting depth also dramatically affects plant performance, particularly under certain circumstances. Shallow roots, which may have relatively low access to available water under drought conditions (Uga et al., 2013), as well as low wind resistance and lodging resistance (Figure 2), can nonetheless increase the acquisition of certain nutrients in topsoil layer (Wang et al., 2010; Lynch, 2011), and they may even increase yield in saline soils by modulating gravitropic responses when roots grow on the soilsurface (Kitomi et al., 2020). The architecture of both shoots and roots plays a determinant role in the performance of crops and other economic plants; hence, the importance of understanding gravitropism for their improvement.

\section{LAZY GENES REGULATE PLANT GRAVITROPISM IN BOTH SHOOT AND ROOT}

Despite much progress having been made in understanding plant gravitropism, there are still many unsolved core questions in the current four-step gravitropism model, particularly the early events spanning from gravity perception to signal transduction (Su et al., 2017). For decades, the starch-statolith theory has been popular for explaining the initial process of gravitropism; there is evidence indicating it may not apply to all species (Edelmann, 2018; Richter et al., 2019; Ditengou et al., 2020). Thus, identifying new players involved in these early events would be vital to understanding the molecular architecture characterizing the early steps of plant gravitropism.

In the past decades, the LAZY gene family has been identified as the key regulator in both roots' positive gravitropism and shoots' negative gravitropism. LAZY genes have been designated with other names apart from LAZY, such as NEGATIVE 
A

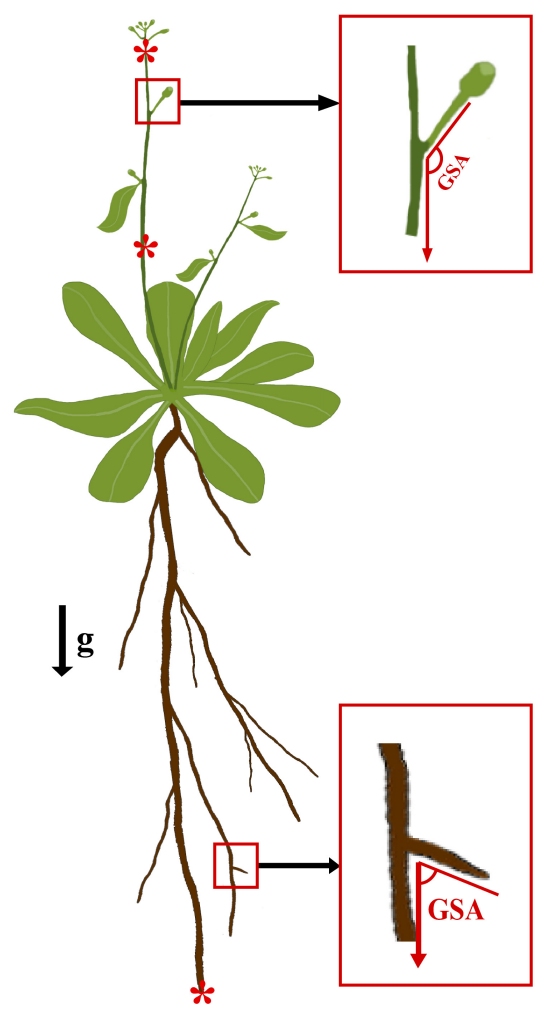

B
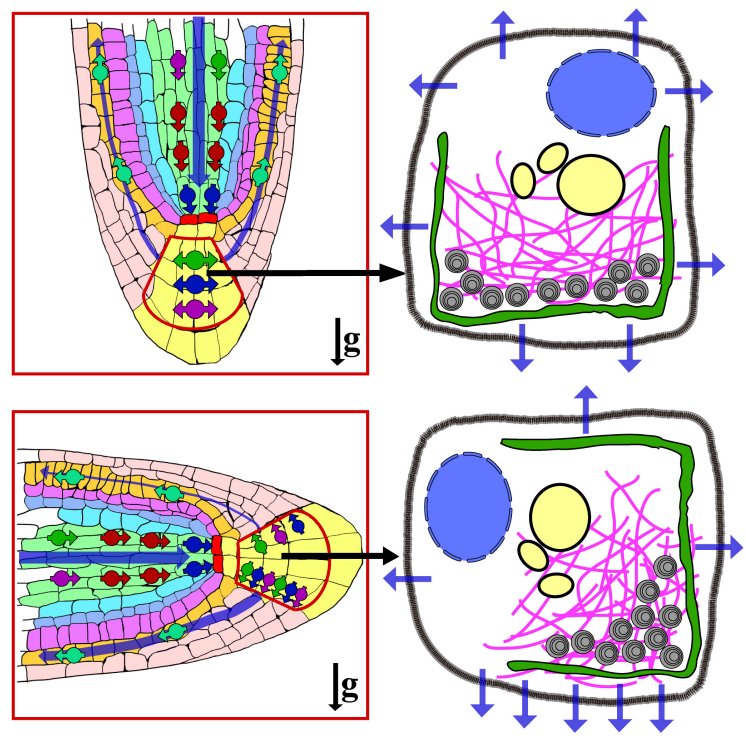

$\square$ Stele
$\square$ Pericycle PIN1

$\square$ Endodermis PIN3

$\square$ Cortex PIN4

$\square$ Epidermis PIN7

$\square$ Columella cell

$\square$ Quiescent center

Lateral root cap

FIGURE 1 | The diagram of GSA and gravitropism of root tip. (A) A plant showing the concept of GSA and gravity-sensing tissues in shoot and root. The asterisks (from top to bottom) show the representative gravity-sensing tissues of inflorescence, shoot, and root, respectively. (B) A schematic showing the activity of PINs and auxin flow in a root tip before (top) and after (bottom) a $90^{\circ}$ reorientation. After a gravistimulation by the $90^{\circ}$ reorientation, the PINs within the columella cell are polarized along with the direction of the gravity, and auxin is asymmetrically transported to the bottom side of the root tip. The arrows of $g$ indicate the direction of gravity vector.
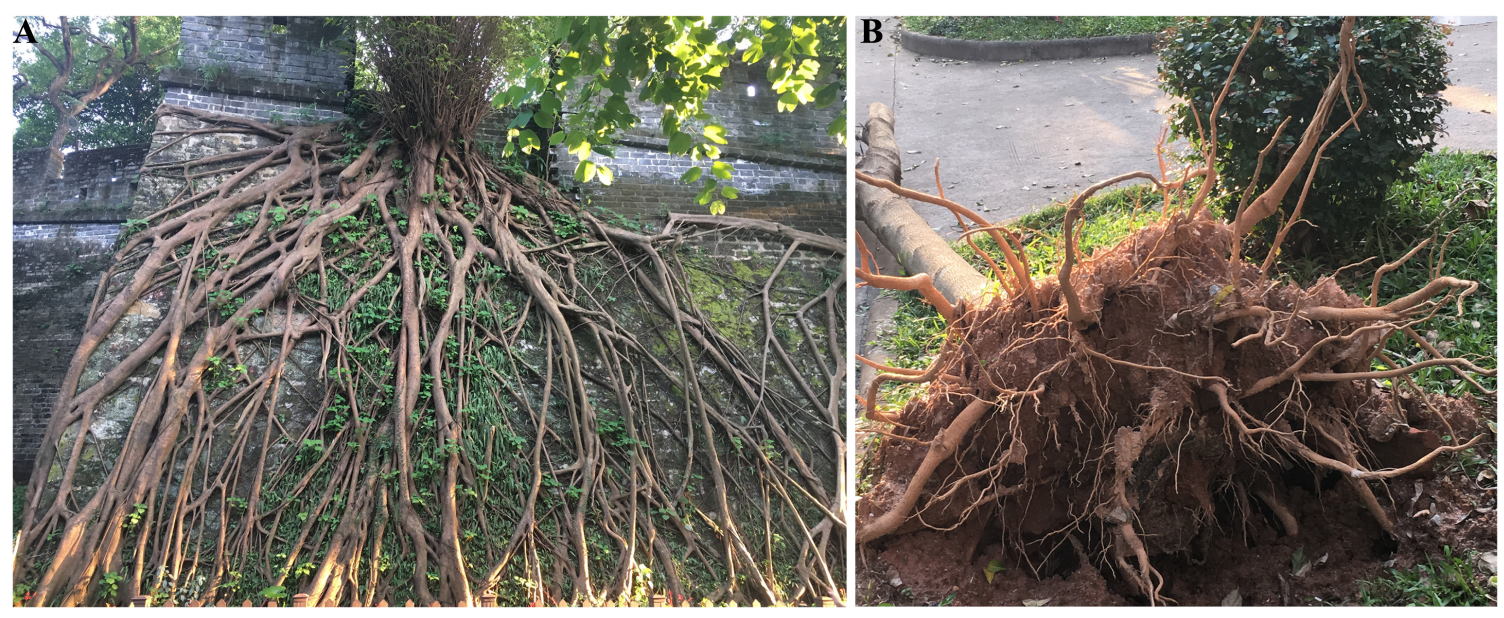

FIGURE 2 | Deep roots and shallow roots. (A) A tree with deep roots on the ancient city wall in Guangzhou Yuexiu Park. (B) A tree with shallow roots that was toppled by the strong winds of "Typhoon Mangkhut." 
GRAVITROPIC RESPONSE OF ROOTS (NGR), and DEEPER ROOTING (DRO). A previous review suggested using $L Z Y$ as the representative nomenclature (Nakamura et al., 2019), so hereon we refer to $L Z Y$ for consistency.

The name lzy originates from those classical rice and maize plants deemed "lazy," which developed spreading tillers in rice or prostate culms in maize (Overbeek, 1936; Jones and Adair, 1938). Phenotypic characterization and physiological studies indicated that the negative gravitropic response of the aboveground shoot of these plants had been severely impaired (Li et al., 2007; Yoshihara and Iino, 2007; Dong et al., 2013). Upon gravitational pull, the horizontally placed hypocotyl, coleoptile, and inflorescence of the $l z y 1$ plants bent more slowly than did their counterparts in wild-type plants. In this way, the shoot grew horizontally or with a large spreading angle under gravitational pull (Overbeek, 1936; Jones and Adair, 1938; Li et al., 2007; Dong et al., 2013). The $l z y 1$ mutant was also identified in Arabidopsis and designated as atlzy1; its branching has outward orientation and wider angles than do the branches of the wild type (Yoshihara et al., 2013; Figure 3A). This growth habit resembles the rice $l z y 1$ mutant and was caused by impaired gravitropic responses, which also led to the slowed dynamics of gravitational curvature of the horizontally placed atlzy1 plants.

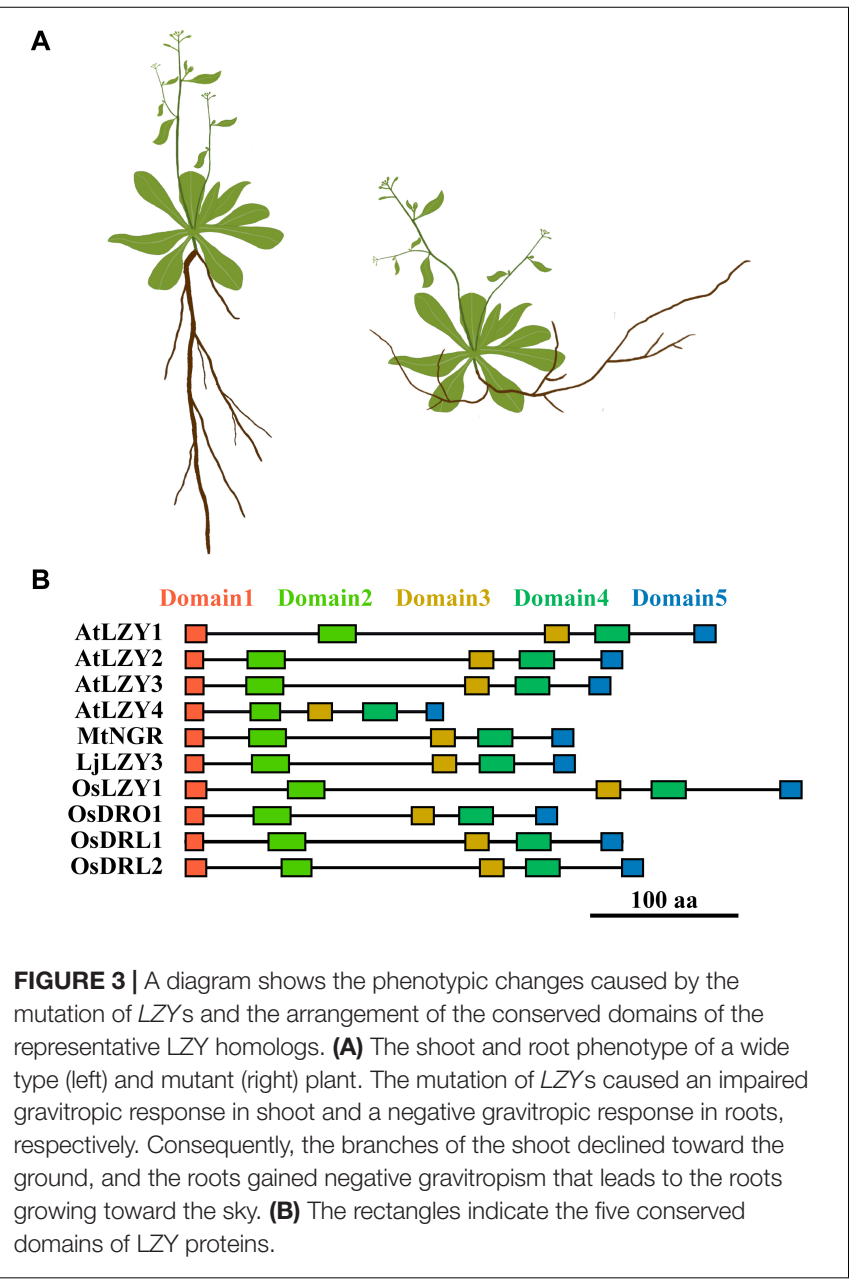

Although root gravitropism functioned normally in the lzyl mutants of rice, maize, and Arabidopsis, a few recent reports have shown that members of the $L Z Y$ gene family are also crucial regulators of the roots' positive gravitropism in several species (Uga et al., 2013; Ge and Chen, 2016; Taniguchi et al., 2017; Yoshihara and Spalding, 2017; Ge and Chen, 2019; Chen et al., 2020; Figure 3A). Disruption of one of the LZY homologs in two legume species, Medicago truncatula and Lotus japonicus reversed the positive gravitropism of their roots to negative gravitropism, resulting in roots that grew upward and emerged above the soil surface (Ge and Chen, 2016; Chen et al., 2020). Additionally, simultaneous mutation of three LZY genes in Arabidopsis also led to upwardly growing roots, clearly a negative gravitropic response, thus resembling the mutants identified in M. truncatula and L. japonicus (Ge and Chen, 2016; Figure 3A). The rice genome carries four LZYs, namely, LZY1, DRO1, DRO1-like 1 (qSOR1, DRL1), and DRO1-like 2 (DRL2) (Ge and Chen, 2016; Kitomi et al., 2020; Table 1). Natural mutation of DRO1 and DRL1, and a knockout mutation of DRL2 by CRISPR/Cas9 technology all led to impaired root gravitropic responses and an altered RSA, even with soil-surface roots (SOR) (Kitomi et al., 2020).

Altogether, these findings strongly demonstrated the key involvement of LZYS in both shoot and root gravitropism. Notably, though functions can be altered by the mutation of LZYs, their outcomes differ in plants. In the aboveground shoots, mutation of LZY1 impairs or weakens shoots' negative gravitropic responses, whereas in roots, the positive gravitropism was reversed into negative gravitropism via mutation of LZY homologs.

\section{LZY PROTEINS REGULATE PAT AND ASYMMETRIC AUXIN DISTRIBUTION}

Intriguingly, the sedimentation of amyloplasts in the abovereported lzy mutants was all normal (Abe et al., 1994; Li et al., 2007; Ge and Chen, 2016; Taniguchi et al., 2017), whereas PAT was dramatically altered in them. In both Arabidopsis atlzy2,3,4 and L. japonicus ljlzy3 mutants, whose roots grew upward, the asymmetrical auxin distribution in the elongation zone of these mutated roots has been completely reversed, leading to more auxin accumulation in the new top flank upon gravistimulation; this contrasts starkly with the wild-type roots, in which the higher accumulation of auxin was found in the new bottom flank (Ge and Chen, 2016; Taniguchi et al., 2017; Yoshihara and Spalding, 2017; Chen et al., 2020). Also, the auxin efflux carrier PINFORMED 3 (PIN3) which is located in the columella cells and is capable of polarizing to redirect auxin flux upon gravistimulation, was reversely polarized in the Arabidopsis atlzy2,3,4 mutant. Moreover, accumulation of the PIN2 protein, which is located in the epidermal and cortex cells of the root tip and crucial for asymmetric auxin flow of root gravitropism was found predominantly at the top flank of ljlzy3 mutant, the opposite of that in the wild-type roots (Chen et al., 2020).

Likewise, the asymmetrical auxin distribution was also severely impaired in the coleoptiles of rice $l z y 1$ and abolished 
TABLE 1 | LZYs in Arabidopsis (A. thaliana), soybean (G. max), lotus (L. japonicus), Medicago (M. truncatula), and rice (O. sativa).

\begin{tabular}{|c|c|c|c|c|c|}
\hline Gene Name & Locus & Species & Similarest Arabidopsis Gene & $E$-value & References \\
\hline AtLZY1 & AT5G14090 & A. thaliana & & & Yoshihara et al., 2013 \\
\hline AtLZY2 & AT1G17400 & A. thaliana & & & Yoshihara et al., 2013; Ge and Chen, 2016 \\
\hline AtLZY3 & AT1G72490 & A. thaliana & & & Yoshihara et al., 2013; Ge and Chen, 2016 \\
\hline AtLZY4 & AT1G19115 & A. thaliana & & & Yoshihara et al., 2013; Ge and Chen, 2016 \\
\hline AtLZY5 & AT3G24750 & A. thaliana & & & Yoshihara et al., 2013 \\
\hline AtLZYG & AT3G27025 & A. thaliana & & & Yoshihara et al., 2013 \\
\hline$G m L Z Y 1$ & Glyma.02G097100 & G. $\max$ & AtLZY1 & $1.43 \mathrm{E}-50$ & This study \\
\hline $\mathrm{GmLZY2}$ & Glyma.10G298100 & G. $\max$ & AtLZY1 & $9.34 \mathrm{E}-43$ & This study \\
\hline $\mathrm{GmLZY3}$ & Glyma.16G054300 & G. $\max$ & AtLZY1 & $1.99 E-54$ & This study \\
\hline $\mathrm{GmLZY4}$ & Glyma.18G284400 & G. $\max$ & AtLZY1 & 3.37E-51 & This study \\
\hline $\mathrm{GmLZY5}$ & Glyma.19G094600 & G. $\max$ & AtLZY1 & $5.22 \mathrm{E}-55$ & This study \\
\hline$G m L Z Y 6$ & Glyma.20G249200 & G. $\max$ & AtLZY1 & $3.5 E-41$ & This study \\
\hline $\mathrm{GmLZY7}$ & Glyma.03G264200 & G. $\max$ & AtLZY3 & $2.95 E-81$ & Ge and Chen, 2016 \\
\hline GmLZY8 & Glyma.07G040800 & G. $\max$ & AtLZY3 & $5.82 E-96$ & Ge and Chen, 2016 \\
\hline$G m L Z Y 9$ & Glyma.09G071100 & G. $\max$ & AtLZY3 & 9.96E-35 & This study \\
\hline GmLZY10 & Glyma.15G179200 & G. $\max$ & AtLZY3 & $1.72 \mathrm{E}-33$ & This study \\
\hline GmLZY11 & Glyma.16G009400 & G. $\max$ & AtLZY3 & 1.59E-96 & Ge and Chen, 2016 \\
\hline GmLZY12 & Glyma.19G263200 & G. $\max$ & AtLZY3 & $3.02 E-86$ & Ge and Chen, 2016 \\
\hline$G m L Z Y 13$ & Glyma.08G356200 & G. $\max$ & AtLZY5 & $5.12 \mathrm{E}-17$ & This study \\
\hline$G m L Z Y 14$ & Glyma.16G094000 & G. $\max$ & AtLZY5 & $7.59 \mathrm{E}-10$ & This study \\
\hline GmLZY15 & Glyma.18G173300 & G. $\max$ & AtLZY5 & $5.73 E-16$ & This study \\
\hline$L j L Z Y 1$ & Lj1g3v3171170 & L. japonicus & AtLZY1 & $2 \mathrm{E}-50$ & Chen et al., 2020 \\
\hline$L j L Z Y 2$ & Lj1g3v3466030 & L. japonicus & AtLZY5 & $2 \mathrm{E}-16$ & Chen et al., 2020 \\
\hline$L j L Z Y 3$ & Lj3g3v2576600 & L. japonicus & AtLZY3 & $9 \mathrm{E}-79$ & Ge and Chen, 2016; Chen et al., 2020 \\
\hline$L j L Z Y 4$ & Lj6g3v1177230 & L. japonicus & AtLZY2 & $2 \mathrm{E}-28$ & Chen et al., 2020 \\
\hline LjLZY5 & Lj0g3v0142099 & L. japonicus & AtLZY5 & $2 \mathrm{E}-10$ & Chen et al., 2020 \\
\hline$L j L Z Y 6$ & Lj0g3v0338439 & L. japonicus & AtLZY1 & $2 \mathrm{E}-25$ & Chen et al., 2020 \\
\hline MtLZY7 & MtrunA17_Chr2g0301041 & M. truncatula & AtLZY2 & 4.27E-09 & This study \\
\hline$M t L Z Y 3$ & MtrunA17_Chr3g0122881 & M. truncatula & AtLZY1 & $1.91 E-43$ & This study \\
\hline$M t L Z Y 2$ & MtrunA17_Chr7g0222391 & M. truncatula & AtLZY1 & $1.24 \mathrm{E}-45$ & This study \\
\hline MtLZY4 & MtrunA17_Chr7g0224671 & M. truncatula & AtLZY1 & $1.31 \mathrm{E}-26$ & This study \\
\hline MtLZY1 & MtrunA17_Chr7g0224731 & M. truncatula & AtLZY1 & $3.4 \mathrm{E}-56$ & This study \\
\hline MtLZY6 & MtrunA17_Chr7g0237671 & M. truncatula & AtLZY5 & $8.2 \mathrm{E}-19$ & This study \\
\hline MtLZY5 & MtrunA17_Chr7g0240781 & M. truncatula & AtLZY5 & 9.09E-20 & This study \\
\hline MtNGR & MtrunA17_Chr8g0344431 & M. truncatula & AtLZY3 & 4.7E-98 & Ge and Chen, 2016 \\
\hline OsDRO1 & LOC_Os09g26840 & O. sativa & AtLZY3 & $6.11 \mathrm{E}-35$ & Uga et al., 2013; Ge and Chen, 2016 \\
\hline OsLZY1 & LOC_Os11g29840 & O. sativa & AtLZY1 & $2.48 \mathrm{E}-15$ & Li et al., 2007 \\
\hline OsDRL2 & LOC_Os03g29270 & O. sativa & AtLZY3 & $3.43 E-29$ & Ge and Chen, 2016; Kitomi et al., 2020 \\
\hline OsDRL1 & LOC_Os07g42290 & O. sativa & AtLZY3 & 4.99E-39 & Ge and Chen, 2016; Kitomi et al., 2020 \\
\hline
\end{tabular}

in the coleoptiles of maize la1-ref mutant after incurring gravistimulation (Yoshihara and Iino, 2007; Dong et al., 2013). Additionally, the basipetal auxin transport in the coleoptiles of both rice lzy1 and maize la1-ref mutant was strongly enhanced ( $\mathrm{Li}$ et al., 2007; Yoshihara and Iino, 2007; Dong et al., 2013), indicating that PAT was altered somehow in $l z y 1$ mutants. Taken together, these findings from both shoots and roots consistently demonstrate that LZYs do not interfere with amyloplasts sedimentation, but rather play a key role in directing asymmetrical auxin distribution upon gravistimulation.

Asymmetrical auxin distribution relies on the polarization of auxin transporters, which is presumably able to trigger an asymmetrical auxin flux, thereby leading to further asymmetry in auxin accumulation (Dhonukshe et al., 2010). Thus, upon gravistimulation, transcytosis-based polarization of the auxin efflux carriers PIN3 and PIN7 in columella cells is essential for roots' positive gravitropism (Dhonukshe et al., 2010). In Arabidopsis, mutation of $L Z Y$ s reversed the polarization of PIN3 onto the new upper side of columella cells (Taniguchi et al., 2017; Ge and Chen, 2019). Moreover, disruption of PINs expressed in columella cells impaired the negative gravitropism of these mutants (Ge and Chen, 2019). These observations align well with the reversed asymmetry of auxin distribution and the negative gravitropic response of mutant roots, which indicates that LZYs regulate auxin distribution by regulating PIN polarization. Thus, LZYs can be defined as being important PINs regulators, directly 
or indirectly, which function to ensure PINs translocate to the correct side upon gravistimulation (Li et al., 2007).

\section{CONSERVED DOMAINS IN LZY PROTEINS}

Although the overall similarity of LZY homologs is relatively low, LZYs proteins do share conserved molecular function. For example, the shared sequence identity between AtLZY1 and AtLZY2 is only $19.8 \%$, yet AtLZY2 was still able to rescue the phenotype of atlzy1 when driven by the promoter of AtLZY1 (Yoshihara and Spalding, 2020). Perhaps this is possible because the LZYs share five conserved domains (Dardick et al., 2013; Yoshihara et al., 2013; Figure 3B), which seem to be decisive for the proper functioning of LZY proteins.

What are the exact functions of these five conserved domains? A recent study has thoroughly investigated the functions of the domains of AtLZY1 by mutating the amino acids. The domain I, which is located at the far $\mathrm{N}$ terminus of the protein and necessary for the plasma membrane localization of AtLZY1, appears to be vital for its function in regulating shoot branch angles. The domain II, harboring the highly conserved signature sequence of the family (Dardick et al., 2013; Yoshihara and Spalding, 2020), is crucial to the protein's function. Once the conserved residues were mutated in Arabidopsis, the auxin gradient was reversed, such that the plant inflorescence gained positive gravitropism, showing the weeping shoot phenotype (Yoshihara and Spalding, 2020). The functions of domain III and IV are still unclear, as their mutation did not change either the subcellular localization or the function of AtLZY1 (Yoshihara and Spalding, 2020).

Domain V, located at the far $\mathrm{C}$ terminus of the proteins, contains an EAR-like motif (Dardick et al., 2013; Yoshihara et al., 2013; Ashraf et al., 2019), and has been termed a conserved C terminus in LZY (CCL) family proteins (Taniguchi et al., 2017) or a WIKTD motif (Dardick et al., 2013). This domain $\mathrm{V}$ is critical for the biological functioning of the protein. When mutated, the normal function of the protein was severely impaired, resulting in the failed rescue of the atlzy1, though the subcellular localization of the protein was unaffected (Yoshihara and Spalding, 2020). According to two recent reports, domain $\mathrm{V}$ can interact with the BREVIS RADIX (BRX) domain, which exists in the plant-specific BRX gene family and PRAF (PH, RCC1, and FYVE)-like family proteins, to mediate homotypic and heterotypic protein interactions (Briggs et al., 2006; Li et al., 2019; Furutani et al., 2020).

The presence of the EAR-like motif in domain $\mathrm{V}$ is rather interesting. The EAR motif can recruit the transcription suppressor TOPLESS (TPL) to suppress the expression of downstream genes via epigenetic regulation, and it is usually found among the transcription factors. A recent report has shown that this domain from wheat LZY homologs can interact with TPL in yeast two-hybrid and bimolecular fluorescence complementation (BiFC) assays (Ashraf et al., 2019), raising the possibility that the mechanic action of LZY family proteins might have a connection with TPL gene family and involves transcriptional regulation.
Overall, a few reports have revealed the role of domain $\mathrm{V}$ from various aspects, which undoubtedly supports its critical contribution to determining the function of LZY proteins, from their subcellular localization to the interaction with partner proteins, and even potential transcriptional regulation. Further analysis of the functioning of domain $\mathrm{V}$ in planta would help to answer questions of how exactly it functions and whether it is indeed actively involved in transcriptional regulation.

\section{SUBCELLULAR LOCALIZATION OF LZY PROTEINS}

Subcellular localization of a protein is an essential piece of information to understand its molecular mechanism. Despite many efforts, the subcellular localization of LZY proteins is still not fully known. The major problem lies in their controversial localization pattern, i.e., plasma membrane versus nucleus. Protein subcellular prediction has found both transmembrane domain and nuclear localization signal (NLS) peptides in OsLZY1 and AtLZY1 (Li et al., 2007; Yoshihara et al., 2013). Whereas a transmembrane domain is a symbol of membrane protein, the NLS and EAR motif are typically found in transcription factors. Transient expression of LZYs fused with the green fluorescence protein (GFP) demonstrated that OsLZY1 and AtLZY1 are localized to both the plasma membrane and nucleus (Li et al., 2007; Yoshihara et al., 2013). Inducible expression of GFP-fused AtLZY1 in transgenic Arabidopsis also showed that AtLZY1 was localized to both the plasma membrane and nucleus (Li et al., 2007). Additionally, disruption of the NLS eliminated the nuclear localization (Li et al., 2007; Yoshihara et al., 2013), and deletion of the transmembrane domain removed the plasma membrane localization (Li et al., 2007). These findings collectively provide convincing evidences that OsLZY1 and AtLZY1 are localized to the plasma membrane and nucleus, though the underlying mechanism likely differs between them. The nucleus localization of AtLZY1 seemed inconsequential (Yoshihara et al., 2013), whereas that of OsLZY1 was clearly essential to its functioning (Li et al., 2019).

OsLZY1 interacts with a member of the rice Brevis Radix family, OsBRX Like 4 (OsBRXL4), on the plasma membrane. This interaction between OsLZY1 and OsBRXL4 was capable of affecting the nucleus localization of OsLZY1. Once the abundance of OsBRXL4 was increased by overexpressing, more OsLZY1 appeared on the plasma membrane with comparatively less found in the nucleus, and the transgenic plant displayed a broader tiller angle (Li et al., 2019). While proteins that localize to both plasma membrane and nucleus are not very common, there is a group of transcription factors, called membrane-bound transcription factors (MTFs), which sense the signal on the membrane and move into the nucleus to exert their regulatory function (Chen et al., 2008; Li et al., 2016). The dynamic partitioning of OsLZY1 protein between the plasma membrane and nucleus upon gravistimulation represents a new segment of the model addressing the role of OsLZY1 in regulating the tiller angles and shoot gravitropism in rice. 
The subcellular localization of many other LZY homologs has also been investigated. These homologs, including AtLZY2, AtLZY3, AtLZY4, and OsDRL1, were mainly localized to the plasma membrane in transient expression assays using Nicotiana benthamiana leaf epidermal cells or protoplast cells (Taniguchi et al., 2017; Ge and Chen, 2019; Chen et al., 2020; Kitomi et al., 2020; Yoshihara and Spalding, 2020). The plasma membrane localization of LZYs lends support to the view that LZYs can regulate the polarization of PINs. However, these findings of subcellular localization were acquired from either transient expression or a snapshot of GFP-fused LZYs. Rather, direct observation of LZYs' subcellular localization in statocyte cells is essential for understanding its mechanism (Nakamura et al., 2019). Unfortunately, fluorescence was always undetectable in the stable transgenic lines expressing the FP-fused LZYs. By observing the fixed and cleared roots, AtLZY3 was found to localize to the plasma membrane of columella cells. Further, the immunolocalization of eGFP-LjLZY3 in L. japonicus showed that LjLZY3, which controls the positive root gravitropism in L. japonicus, predominantly localized to the basal and apical sides of the plasma membrane in root stele cells (Chen et al., 2020).

Despite many reports consistently supporting the view that LZY homologs controlling root gravitropism are localized to the plasma membrane, other findings have indicated their nucleus localization. Whereas the full-length OsDRO1, which is encoded by a quantitative trait locus (QTL), was localized to the plasma membrane, the truncated form of OsDRO1 was localized to the plasma membrane and cytoplasm, as well as the nucleus (Uga et al., 2013). By fusing it to the rapidly maturing VENUS, Waite et al. (2020) recently found that AtLZY3 was largely localized to the nucleus of the cortical and endodermal cells of both primary and lateral root tips in the stable transgenic lines under the native conditions. Unfortunately, the fluorescence signal of this AtLZY3-VENUS still went undetected in the columella cells, implying that AtLZY3's subcellular localization might be conditional and potentially reliant on cell type. Further investigation into the dynamic subcellular localization of LZY homologs in columella cells under native condition upon gravistimulation is now warranted, as this would provide more valuable information to address the detailed molecular mechanism of LZYs.

\section{MECHANISM OF LZYS CONTROLLING SHOOT AND ROOT GRAVITROPISM}

Studies with mutants have revealed that LZYs control the gravitropism of shoots and roots by directing auxin flow. However, the underlying mechanism is not yet fully understood. Recently, studies of OsLZY1 and AtLZY3 have shed light on the molecular mechanism by which LZY proteins could control the plant gravitropism process.

As discussed above, the $\mathrm{C}$ terminus is highly conserved among the LZY gene family. The studies using rice and Arabidopsis have both found that this $\mathrm{C}$ terminus, which harbors the EARLike motif, interacts with the BRX domains (Li et al., 2019; Furutani et al., 2020). In rice, OsLZY1 interacts with OsBRXL4 on the plasma membrane, where they are co-localized (Li et al., 2019). The interaction between OsLZY1 and OsBRXL4 was likely to retain OsLZY1 on the plasma-membrane, further altering the relative proportions of membrane-localized and nucleuslocalized OsLZY1 proteins (Li et al., 2019; Figure 4A). Since nucleus localization is critical for OsLZY1 to exert its function, the interaction between it and OsBRXL4 profoundly influences the function of OsLZY1 and, by extension, the rice tiller angle (Li et al., 2019). When OsBRXL4 was overexpressed, OsLZY1 was less likely to be localized to the nucleus, resulting in prostrate growth of rice tillers, which mimics the phenotype of the oslzy1 mutant. By contrast, in the RNA interference (RNAi) lines, in which multiple OsBRXL homologs were targeted, OsLZY1 was more likely to be localized to the plasma membrane, resulting in more compact shoot architecture (Li et al., 2019). These results indicated that the balanced dynamic subcellular localization of OsLZY1 between the nucleus and plasmamembrane is crucial for determining its function and thus rice shoot architecture (Figure 4A).

Recently, AtLZY3 was also reported to interact with the BRX domain of Regulator of Chromosome Condensation 1 (RCC1)like domain (RLD) proteins (Furutani et al., 2020). By imaging the roots that have been fixed and cleared, AtLZY3 was found

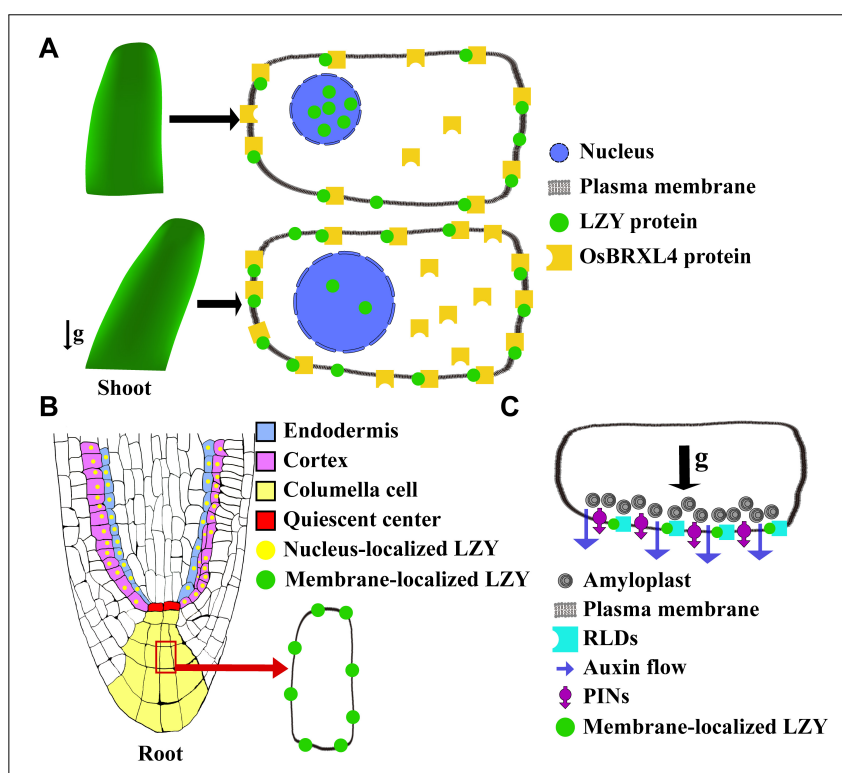

FIGURE 4 | Diagrams illustrating the function of LZY proteins in regulating plant gravitropism. (A) In the shoot of rice, OsLZY1 interacts with OsBRXL4 to regulate shoot gravitropism. OsLZY1 is localized to both the plasma membrane and nucleus. The nuclear localization of OSLZY1 is important for its function. OsBRXL4, which bears three BRX domains and physically interacts with OsLZY1 at the plasma membrane, can regulate the proportions of membrane-localized and nucleus-localized OsLZY1 proteins. In case that OsBRXL4 is increased, the nucleus-localized OsLZY1 reduces, leading to a wider tiller angle (bottom). (B) The $L Z Y$ s are expressed in the root-tip columella cells. The protein of AtLZY3 was also detected in the cortex and endodermis cells. (C) LZYs interact with RLDs, which bear one BRX domain, at the plasma membrane. After gravistimulation by a $90^{\circ}$ reorientation, LZYs, RLDs, and PINs polarize along with the direction of the gravity to the bottom-side membrane of the columella cells, which further triggers PAT. 
localized to the plasma membrane of columella cells. Upon gravistimulation, AtLZY3 polarized to the new bottom side columella cells and recruited RLDs onto the plasma membrane by interacting with them via the $\mathrm{C}$ terminus (i.e., the domain $\mathrm{V})$, thereby triggering relocalization of PIN3 and a downstream reaction (Furutani et al., 2020; Figures 4B,C). RLDs have also been called PRAF family proteins (Briggs et al., 2006). Besides the tandem repeat of the RCC1 domain at the middle and the BRX domain at the $\mathrm{C}$ terminus, RLDs also harbor two other conserved domains: the pleckstrin homology $(\mathrm{PH})$ domain at the $\mathrm{N}$ terminus, and the FYVE zinc-finger domain situated between the RCC1 and BRX domains (Briggs et al., 2006; Wywial and Singh, 2010). RLDs appear to regulate auxin transport by modulating PINs' expression and localization (Furutani et al., 2020). Thus, AtLZY3 regulates auxin transport and root gravitropism by recruiting RLDs to the plasma membrane to regulate PINs' relocalization (Figures 4B,C). It is noteworthy that the FYVE domain is known for its function in membrane trafficking ( $\mathrm{Hu}$ et al., 2002). Thus, it is plausible that RLDs-LZYs might represent a pathway operating to regulate PINs' polarization by a novel type of vesicle trafficking mechanism.

As discussed above, the LZYs' subcellular localization is a substantial component of the molecular mechanism underpinning LZYs' activity in gravitropism. Given the current reports that LZYs may demonstrate varied subcellular localizations depending on the cell type and conditions, more investigation of the dynamic localization of AtLZY3 and other LZY homologs in both statocyte cells and other tissues will help to uncover the subcellular localization profile and mechanism of the LZYs that control root gravitropism. Nevertheless, the finding that LZYs interact with BRX domain-containing proteins to regulate auxin transport is a monumental step forward in elucidating the mechanism by which LZYs control plant gravitropism, albeit one that could be species-specific.

\section{FUNCTIONAL DIVERGENCE OF THE LZY FAMILY}

LZY genes have tended to form a small gene family in higher plants, such as Arabidopsis, rice, M. truncatula, and L. japonicus (Yoshihara et al., 2013; Nakamura et al., 2019; Figure 5 and Table 1). In Arabidopsis, there are six members in the gene family. Since the function of AtLZY5 and AtLZY6 in gravitropism has not been studied yet, we will not discuss them and their homologs in other species. According to mutant studies and our phylogenetic analysis (Figure 5), the LZYs in Arabidopsis have diverged in their functioning across varied organs. Specifically, AtLZY1 regulates shoot and inflorescence stem gravitropism, whereas AtLZY4 regulates root gravitropism. AtLZY2 and AtLZY3, however, regulate both shoot and root gravitropism (Yoshihara et al., 2013; Ge and Chen, 2016; Taniguchi et al., 2017; Yoshihara and Spalding, 2017; Nakamura et al., 2019). The synteny analysis indicated AtLZY2, AtLZY3, and AtLZY4 are located within the micro-syntenic blocks on chromosome 1 (Ge and Chen, 2016). It is known that the Arabidopsis genome

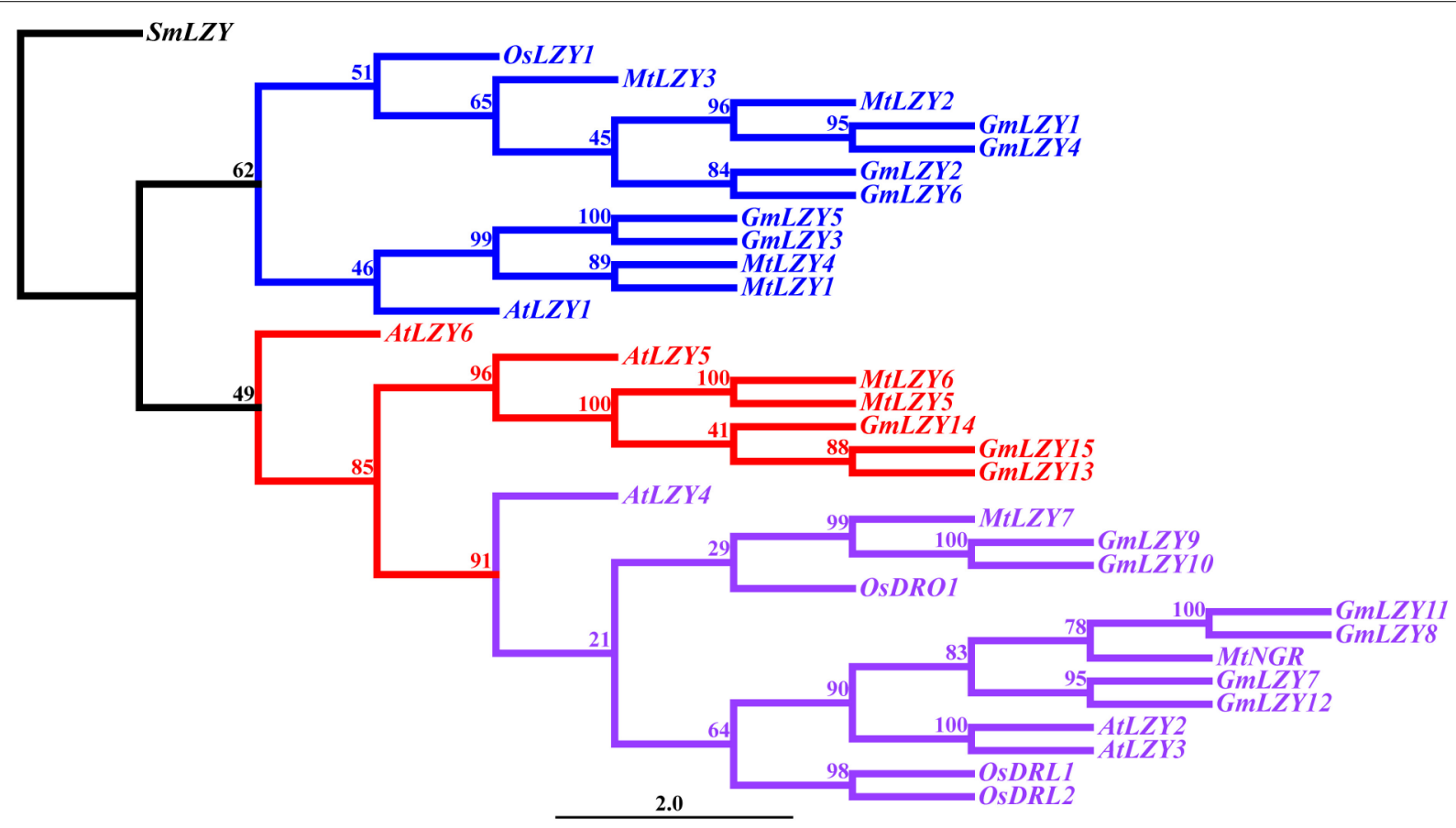

FIGURE 5 | Phylogenetic analysis of LZYs among different species. The phylogenetic tree was reconstructed using the maximum-likelihood method with 1,000 bootstrap repeats. Blue represents the clade that mainly regulates shoot gravitropism. Purple represents the DRO1 clade. Red represents the clade with a currently unknown function. SmLZY served as the outgroup when constructing the tree. The numbers on the branches indicate the bootstrap values. Sm, Selaginella moellendorffii; Os, Oryza sativa; Mt, Medicago truncatula; Gm, Glycine max; At, Arabidopsis thaliana. 
has undergone three whole-genome duplications (WGD) events in its evolution, resulting in $60 \%$ of genes possessing paralogs in the corresponding syntenic fragments (Bowers et al., 2003; Panchy et al., 2016). The syntenic relationships of AtLZY2, AtLZY3, and AtLZY4 suggest their three LZYs are derived from the WGD events of Arabidopsis. The divergence of LZYs in regulating gravitropism of different organs seems correlated with their spatial expression pattern, in that AtLZY1 and AtLZY4 are expressed mainly in shoots and roots, respectively, whereas AtLZY2 and AtLZY3 are expressed in both shoot and root. In a recent study, expression pattern divergence of gene pairs derived from WGD was observed in Arabidopsis (De Smet et al., 2017), thus explaining the divergence of the LZYs.

The $L Z Y$ s in rice also displayed evidence of functional divergence. OsLZY1 controls the tiller angle by regulating shoot gravitropism (Li et al., 2007; Yoshihara and Iino, 2007; Li et al., 2019), whereas OsDRO1, OsDRL1, and OsDRL2, which were phylogenetically clustered into the DRO1 group (Hollender and Dardick, 2015; Figure 5), regulate the root gravitropic response and hence the growth angle of both seminal and crown roots (Uga et al., 2013; Kitomi et al., 2020). The lzy mutants with negative gravitropic responses in their roots were also identified in the two legume species M. truncatula and L. japonicus (Ge and Chen, 2016; Chen et al., 2020). These two legume mutants resembled the atlzy2/atlzy3/atlzy4 triple mutant of Arabidopsis (Ge and Chen, 2016; Chen et al., 2020). Notably, the causative genes of these legume mutants lie within the syntenic genome fragments, which are also syntenic to those genome blocks harboring AtLZY2, AtLZY3, AtLZY4, and OsDRO1 (Ge and Chen, 2016). According to the synteny analysis, OsDRO1, OsDRL1, and OsDRL2 are paralogs, having a similar function in regulating root gravitropism in rice (Ge and Chen, 2016; Kitomi et al., 2020). It is possible that knocking-out these three paralogs may strongly enhance the gravitropic defect observed in the near-isogenic lines (Uga et al., 2013; Kitomi et al., 2020), or even completely reverse the positive gravitropism to negative gravitropism in roots, thereby resembling the legume root mutants and Arabidopsis atlzy2/atlzy3/atlzy4 triple mutants.

Interestingly, both OsDRO1 and OsDRL1 are QTLs harboring natural variation among rice populations, and this contributes to the varied RSA in cultivated rice, suggesting that variation in LZY genes may strongly affect rooting depth and plant rooting strategies (Uga et al., 2013). As discussed previously, rooting depth is a fundamental element of the diverse RSA and it dramatically influences a crops' performance. Variation in LZYs can lead to varied gravitropic responses of roots, which further changes the GSA of the root tip, resulting in varied rooting depth. Thus, manipulating the activity of LZYs offers a very promising solution to engineering the plant RSA in crops, trees, and other economically important plants.

\section{LZYS BROADLY EXIST IN THE PLANT KINGDOM}

Since the identification of $L Z Y$ genes in several angiosperm plants, including maize, Arabidopsis, M. truncatula, L. japonicus, and Prunus domestica (Dong et al., 2013; Yoshihara et al., 2013; Howard et al., 2014; Ge and Chen, 2016; Taniguchi et al., 2017; Yoshihara and Spalding, 2017; Chen et al., 2020), similaritybased sequences searches have found the presence of LZY homologs broadly in plant genomes, including the primitive plant Physcomitrella patens (moss), Selaginella moellendorffi (fern), and gymnosperm plant Picea sitchensis (spruce) (Dardick et al., 2013; Yoshihara et al., 2013). As discussed above, the overall sequence similarity of these LZYs from various species is low, whereas the identity of the five domains is quite high. These conserved domains have been shown to be critical to the functioning of the proteins (Yoshihara and Spalding, 2020), thus, it is possible that LZYs from different species may share functional conservation to a certain extent. Additionally, all LZY genes from moss to higher plant preserved a very similar intron-exon pattern, particularly the first six base pairs of the coding sequence immediately after the five prime untranslated regions ( $5^{\prime}$ UTR) of the first exon (Dardick et al., 2013). The comparative genomic analysis also found that $L Z Y$ family members are located within the syntenic genome fragments from monocot to dicots (Ge and Chen, 2016). All these findings concur, by extensively demonstrating the high conservation of LZYs, whose corollary is that LZYs share a common evolutionary origin.

\section{LZYS BELONG TO IGT GENE FAMILY}

While mutations of LZY1 genes leads to impaired shoot gravitropism, wider tiller angles, or a prostrate growth habit in diverse species, mutation of Tiller Angle Controller 1 (TAC1) confers a narrower tiller angle in rice, lateral axillary branches in Arabidopsis, and pillar shoot architecture in trees, the opposite phenotypic traits of $l z y 1$ mutants (Yu et al., 2007; Dardick et al., 2013; Hollender et al., 2018). LZY proteins and TAC1 share similar sequence structures and conserved domains, except that TAC1 lacks the critical domain V that contains the EARlike motif. Phylogenetic analysis suggested that LZYs and TAC1 constituted two clades of the IGT gene family, which is named after the conserved unique motif ( $\mathrm{G} \varphi \mathrm{L}(\mathrm{A} / \mathrm{T}) \mathrm{IGT})$ located within the second domain of LZY and the TAC1 gene family (Dardick et al., 2013; Hollender and Dardick, 2015).

Given the similar sequence pattern but opposite consequences arising from mutation of $L Z Y 1$ and TAC1, the relationship between $L Z Y 1$ and TAC1 and the hidden mechanism responsible for the opposite effects is very interesting. Might there be a direct connection or genetic/physical interaction between these two proteins? A recent work has revealed that LZY1 and TAC1 do show a similar expression pattern in shoot tissues in Arabidopsis (Hollender et al., 2020). The double mutant analysis indicated that $l z y 1$ was epistatic to tac1 with respect to the shoot's branch angles and orientation (Hollender et al., 2020). Unlike LZY1, which controls shoot architecture by modulating gravitropism, the shoot of tacl normally responds to gravitation pull (Hollender et al., 2020), implying that the regulatory and interaction mechanism underlying LZY1 and TAC1 is rather complex and goes beyond a pure gravitropic regulation. 
While LZY genes widely exist in terrestrial plants, including primitive taxa, TAC1 was not found in the latter, but it is present in the vascular plants with lateral axillary organs. Thus, it is plausible that TAC1 is evolved from LZY genes as a truncated LZY (Hollender and Dardick, 2015). The emergence of TAC1 in plant genomes appears to be associated with the origination of lateral shoots in plants evolution (Dardick et al., 2013; Hollender and Dardick, 2015). The counterbalancing effects between LZY1 and TAC1 may have provided a delicate mechanism for attaining the diverse shoot architecture seen among higher plants.

\section{CONCLUSION AND PERSPECTIVES}

Due to their sessile nature, plants cannot move away from the microenvironment where they are established. Yet, plants can sensitively detect various environmental signals, such as light, temperature, gravitation pull, and water availability. Beyond that, plants have evolved complex mechanisms, by which they can utilize environmental signals as guides to adjust their growth, physiological status, and even behavior, in a plastic manner, thereby achieving a better fit to the current environment in which they live. In this context, gravity can be viewed as an important plant growth regulator, since plants' fitness depends on orienting their tissues properly along its field.

The process of gravitropism, which is triggered by gravitational pull and results in the bending of plant organs, offers plants better adaptation by guiding their roots to grow deep into the soil to uptake water and nutrients, and steering shoots to grow away above the ground to capture incident sun radiation needed for photosynthesis and reproduction. This process, requiring the redistribution of auxin under the guide of gravity, was an evolutionary milestone, in going from ancestral aquatic life, which existed only in the buoyancy environment, to land plants able to sense and respond to gravitational pull properly. LZY genes are among the essential components of the plant gravitropism chain, since mutations of LZYs result in the failure of proper gravitropic responses, in both shoots and roots. LZYs have been widely found in land plants, including the primitive lineages, but not in the aquatic algal genome (Dardick et al., 2013; Yoshihara et al., 2013); this suggests that the emergence of LZY genes may have contributed to the establishment of gravitropism in land plants, thus conferring to them a better fitness within the gravitational field of the Earth.

Gravitropism is a fascinating subject and a focus of plant science study for centuries. However, the molecular architecture of this highly complex form of tropism is largely murky, particularly concerning the details of signal perception through to asymmetric auxin redistribution (Nakamura et al., 2019). Recent

\section{REFERENCES}

Abe, K., Takahashi, H., and Suge, H. (1994). Localization of cells containing sedimented amyloplasts in the shoots of normal and lazy rice ssteedlings. Uchu Seibutsu Kagaku 8, 221-225. doi: 10.2187/bss.8.221

Ashraf, A., Rehman, O. U., Muzammil, S., Leon, J., Naz, A. A., Rasool, F., et al. (2019). Evolution of Deeper Rooting 1-like homoeologs in wheat entails the research from multiple species has consistently demonstrated that LZYs' mutation alters the auxin transport in the shoot, and reversed auxin asymmetry in the root tip. By identifying an important player in the early events of plant gravitropism process, this body of work has also opened a new doorway to investigate the molecular mechanism triggering the relocation of auxin carriers and corresponding asymmetrical auxin flux.

The subcellular localization of LZYs varies under different experimental conditions, which may imply a naturally dynamic and conditional localization of these proteins, potentially involving the movement of LZYs among certain organelles. The recent finding that AtLZY3 is expressed in endodermal and cortical cells in root tips is intriguing, as it implies an unknown mystery behind LZYs. Subcellular localization and tissue-specific expression pattern are the fundamentals to understand the function of a gene. Thus, future efforts to further address the dynamic subcellular localization and tissue-specific expression pattern of LZYs will undoubtedly strengthen the current picture of LZYs as the key regulators of gravitropism. That both BRXs and RLDs can interact with LZYs represents a remarkable breakthrough in unraveling how LZYs mechanistically control gravitropism, and also represents future directions to explore in further resolving the molecular architecture of plant gravitropism, which possibly involves a new type of vesicle trafficking that regulates the movement of LZYs within cells.

In conclusion, the identification and studies of LZY genes have provided fresh and exciting insight into the mechanism of plant gravitropism; future studies into the genetic and molecular details of the LZY-dependent signaling pathway upon gravistimulation will establish the connection between LZYs and downstream reaction, significantly advancing our understanding of the most mysterious part of plant gravitropism.

\section{AUTHOR CONTRIBUTIONS}

ZJ and LG wrote the manuscript. HD, SC, and WH revised the manuscript. All authors read and approved the manuscript.

\section{FUNDING}

The authors are grateful to the National Natural Science Foundation of China for funding support (NSFC, Project 32070305 and 31771345). This work was also supported by the startup fund provided to Ge's lab by South China Agricultural University.

C-terminus mutations as well as gain and loss of auxin response elements. PLoS One 14:e0214145. doi: 10.1371/journal.pone.0214145

Baldwin, K. L., Strohm, A. K., and Masson, P. H. (2013). Gravity sensing and signal transduction in vascular plant primary roots. Am. J. Bot. 100, 126-142. doi: $10.3732 /$ ajb. 1200318

Blancaflor, E. B. (2013). Regulation of plant gravity sensing and signaling by the actin cytoskeleton. Am. J. Bot. 100, 143-152. doi: 10.3732/ajb.1200283 
Blancaflor, E. B., and Masson, P. H. (2003). Plant gravitropism. Unraveling the ups and downs of a complex process. Plant Physiol. 133, 1677-1690. doi: 10.1104/pp.103.032169

Bowers, J. E., Chapman, B. A., Rong, J., and Paterson, A. H. (2003). Unravelling angiosperm genome evolution by phylogenetic analysis of chromosomal duplication events. Nature 422, 433-438. doi: 10.1038/nature01521

Briggs, G. C., Mouchel, C. F., and Hardtke, C. S. (2006). Characterization of the plant-specific BREVIS RADIX gene family reveals limited genetic redundancy despite high sequence conservation. Plant Physiol. 140, 1306-1316. doi: 10. 1104/pp.105.075382

Chen, Y., Xu, S., Tian, L., Liu, L., Huang, M., Xu, X., et al. (2020). LAZY3 plays a pivotal role in positive root gravitropism in Lotus japonicus. J. Exp. Bot. 71, 168-177. doi: 10.1093/jxb/erz429

Chen, Y. N., Slabaugh, E., and Brandizzi, F. (2008). Membrane-tethered transcription factors in Arabidopsis thaliana: novel regulators in stress response and development. Curr. Opin. Plant Biol. 11, 695-701. doi: 10.1016/j.pbi.2008. 10.005

Dardick, C., Callahan, A., Horn, R., Ruiz, K. B., Zhebentyayeva, T., Hollender, C., et al. (2013). PpeTAC1 promotes the horizontal growth of branches in peach trees and is a member of a functionally conserved gene family found in diverse plants species. Plant J. 75, 618-630. doi: 10.1111/tpj.12234

De Smet, R., Sabaghian, E., Li, Z., Saeys, Y., and Van De Peer, Y. (2017). Coordinated Functional Divergence of Genes after Genome Duplication in Arabidopsis thaliana. Plant Cell 29, 2786-2800. doi: 10.1105/tpc.17.00531

Dhonukshe, P., Huang, F., Galvan-Ampudia, C. S., Mahonen, A. P., Kleine-Vehn, J., Xu, J., et al. (2010). Plasma membrane-bound AGC3 kinases phosphorylate PIN auxin carriers at TPRXS(N/S) motifs to direct apical PIN recycling. Development 137, 3245-3255. doi: 10.1242/dev.052456

Digby, J., and Firn, R. D. (1995). The gravitropic set-point angle (GSA): the identification of an important developmentally controlled variable governing plant architecture. Plant Cell Environ. 18, 1434-1440. doi: 10.1111/j.1365-3040. 1995.tb00205.x

Ditengou, F. A., Teale, W. D., and Palme, K. (2020). Settling for Less: Do Statoliths Modulate Gravity Perception? Plants-Basel 9:121. doi: 10.3390/plants9010121

Dong, Z., Jiang, C., Chen, X., Zhang, T., Ding, L., Song, W., et al. (2013). Maize LAZY1 mediates shoot gravitropism and inflorescence development through regulating auxin transport, auxin signaling, and light response. Plant Physiol 163, 1306-1322. doi: 10.1104/pp.113.227314

Edelmann, H. G. (2018). Graviperception in maize plants: is amyloplast sedimentation a red herring? Protoplasma 255, 1877-1881. doi: 10.1007/ s00709-018-1272-7

Friml, J., Wisniewska, J., Benkova, E., Mendgen, K., and Palme, K. (2002). Lateral relocation of auxin efflux regulator PIN3 mediates tropism in Arabidopsis. Nature 415, 806-809. doi: 10.1038/415806a

Furutani, M., Hirano, Y., Nishimura, T., Nakamura, M., Taniguchi, M., Suzuki, K., et al. (2020). Polar recruitment of RLD by LAZY1-like protein during gravity signaling in root branch angle control. Nat. Commun. 11:76.

Ge, L., and Chen, R. (2016). Negative gravitropism in plant roots. Nat. Plants 2:16155.

Ge, L., and Chen, R. (2019). Negative gravitropic response of roots directs auxin flow to control root gravitropism. Plant Cell Environ. 42, 2372-2383. doi: $10.1111 /$ pce. 13559

Haga, K., and Iino, M. (2006). Asymmetric distribution of auxin correlates with gravitropism and phototropism but not with autostraightening (autotropism) in pea epicotyls. J. Exp. Bot. 57, 837-847. doi: 10.1093/jxb/erj069

Hollender, C. A., and Dardick, C. (2015). Molecular basis of angiosperm tree architecture. N. Phytol. 206, 541-556. doi: 10.1111/nph.13204

Hollender, C. A., Hill, J. L. Jr., Waite, J., and Dardick, C. (2020). Opposing influences of TAC1 and LAZY1 on Lateral Shoot Orientation in Arabidopsis. Sci. Rep. 10:6051.

Hollender, C. A., Waite, J. M., Tabb, A., Raines, D., Chinnithambi, S., and Dardick, C. (2018). Alteration of TAC1 expression in Prunus species leads to pleiotropic shoot phenotypes. Hortic Res. 5:26.

Hou, G., Mohamalawari, D. R., and Blancaflor, E. B. (2003). Enhanced gravitropism of roots with a disrupted cap actin cytoskeleton. Plant Physiol. 131, 1360-1373. doi: 10.1104/pp.014423

Howard, T. P. III, Hayward, A. P., Tordillos, A., Fragoso, C., Moreno, M. A., Tohme, J., et al. (2014). Identification of the maize gravitropism gene lazy plant1 by a transposon-tagging genome resequencing strategy. PLoS One 9:e87053 doi: 10.1371/journal.pone.0087053

Imagawa, K., Toko, K., Ezaki, S., Hayashi, K., and Yamafuji, K. (1991). Electrical Potentials during Gravitropism in Bean Epicotyls. Plant Physiol. 97, 193-196. doi: 10.1104/pp.97.1.193

Hu, Y., Chuang, J. Z., Xu, K., Mcgraw, T. G., and Sung, C. H. (2002). SARA, a FYVE domain protein, affects Rab5-mediated endocytosis. J. Cell Sci. 115, 4755-4763. doi: $10.1242 /$ jcs. 00177

Jones, J. W., and Adair, C. R. (1938). A "lazy" mutation in rice*. J. Heredity 29, 315-318. doi: 10.1093/oxfordjournals.jhered.a104527

Kitomi, Y., Hanzawa, E., Kuya, N., Inoue, H., Hara, N., Kawai, S., et al. (2020). Root angle modifications by the DRO1 homolog improve rice yields in saline paddy fields. Proc. Natl. Acad. Sci. 2020, 2005911117.

Leitz, G., Kang, B. H., Schoenwaelder, M. E., and Staehelin, L. A. (2009). Statolith sedimentation kinetics and force transduction to the cortical endoplasmic reticulum in gravity-sensing Arabidopsis columella cells. Plant Cell 21, 843-860. doi: 10.1105/tpc.108.065052

Li, P., Wang, Y., Qian, Q., Fu, Z., Wang, M., Zeng, D., et al. (2007). LAZY1 controls rice shoot gravitropism through regulating polar auxin transport. Cell Res. 17, 402-410. doi: 10.1038/cr.2007.38

Li, S., Wang, N., Ji, D., Xue, Z., Yu, Y., Jiang, Y., et al. (2016). Evolutionary and Functional Analysis of Membrane-Bound NAC Transcription Factor Genes in Soybean. Plant Physiol. 172, 1804-1820. doi: 10.1104/pp.16.01132

Li, Z., Liang, Y., Yuan, Y., Wang, L., Meng, X., Xiong, G., et al. (2019). OsBRXL4 Regulates Shoot Gravitropism and Rice Tiller Angle through Affecting LAZY1 Nuclear Localization. Mol. Plant 12, 1143-1156. doi: 10.1016/j.molp.2019. 05.014

Luschnig, C., Gaxiola, R. A., Grisafi, P., and Fink, G. R. (1998). EIR1, a root-specific protein involved in auxin transport, is required for gravitropism in Arabidopsis thaliana. Genes Dev. 12, 2175-2187. doi: 10.1101/gad.12.14.2175

Lynch, J. P. (2011). Root phenes for enhanced soil exploration and phosphorus acquisition: tools for future crops. Plant Physiol. 156, 1041-1049. doi: 10.1104/ pp.111.175414

Morita, M. T., and Tasaka, M. (2004). Gravity sensing and signaling. Curr. Opin. Plant Biol. 7, 712-718. doi: 10.1016/j.pbi.2004.09.001

Muller, A., Guan, C., Galweiler, L., Tanzler, P., Huijser, P., Marchant, A., et al. (1998). AtPIN2 defines a locus of Arabidopsis for root gravitropism control. EMBO J. 17, 6903-6911. doi: 10.1093/emboj/17.23.6903

Nakamura, M., Nishimura, T., and Morita, M. T. (2019). Bridging the gap between amyloplasts and directional auxin transport in plant gravitropism. Curr. Opin. Plant Biol. 52:60.

Overbeek, J. V. (1936). LAZY," AN A-GEOTROPIC FORM OF MAIZE: "Gravitational Indifference" Rather Than Structural Weakness Accounts for Prostrate Growth-Habit of This Form. J. Heredity 27, 93-96. doi: 10.1093/ oxfordjournals.jhered.a104191

Panchy, N., Lehti-Shiu, M., and Shiu, S. H. (2016). Evolution of Gene Duplication in Plants. Plant Physiol. 171, 2294-2316.

Richter, P., Strauch, S. M., and Lebert, M. (2019). Disproval of the StarchAmyloplast Hypothesis? Trends Plant Sci. 24, 291-293. doi: 10.1016/j.tplants. 2019.02.008

Rouse, D., Mackay, P., Stirnberg, P., Estelle, M., and Leyser, O. (1998). Changes in auxin response from mutations in an AUX/IAA gene. Science 279, 1371-1373. doi: $10.1126 /$ science.279.5355.1371

Saito, C., Morita, M. T., Kato, T., and Tasaka, M. (2005). Amyloplasts and vacuolar membrane dynamics in the living graviperceptive cell of the Arabidopsis inflorescence stem. Plant Cell 17, 548-558. doi: 10.1105/tpc.104.026138

Su, S. H., Gibbs, N. M., Jancewicz, A. L., and Masson, P. H. (2017). Molecular Mechanisms of Root Gravitropism. Curr. Biol. 27, R964-R972.

Swarup, R., Kramer, E. M., Perry, P., Knox, K., Leyser, H. M., Haseloff, J., et al. (2005). Root gravitropism requires lateral root cap and epidermal cells for transport and response to a mobile auxin signal. Nat. Cell Biol. 7, 1057-1065. doi: $10.1038 /$ ncb1316

Taniguchi, M., Furutani, M., Nishimura, T., Nakamura, M., Fushita, T., Iijima, K., et al. (2017). The Arabidopsis LAZY1 Family Plays a Key Role in Gravity Signaling within Statocytes and in Branch Angle Control of Roots and Shoots. Plant Cell 29, 1984-1999. doi: 10.1105/tpc.16.00575

Uga, Y., Sugimoto, K., Ogawa, S., Rane, J., Ishitani, M., Hara, N., et al. (2013). Control of root system architecture by DEEPER ROOTING 1 increases rice 
yield under drought conditions. Nat. Genet. 45, 1097-1102. doi: 10.1038/ng. 2725

Waite, J. M., Collum, T. D., and Dardick, C. (2020). AtDRO1 is nuclear localized in root tips under native conditions and impacts auxin localization. Plant Mole. Biol. 103:210.

Wang, X., Yan, X., and Liao, H. (2010). Genetic improvement for phosphorus efficiency in soybean: a radical approach. Ann. Bot. 106, 215-222. doi: 10.1093/ aob/mcq029

Wu, X., Tang, D., Li, M., Wang, K., and Cheng, Z. (2013). Loose Plant Architecture1, an INDETERMINATE DOMAIN protein involved in shoot gravitropism, regulates plant architecture in rice. Plant Physiol. 161, 317-329. doi: $10.1104 /$ pp.112.208496

Wywial, E., and Singh, S. M. (2010). Identification and structural characterization of FYVE domain-containing proteins of Arabidopsis thaliana. BMC Plant Biol. 10:157. doi: 10.1186/1471-2229-10-157

Yoshihara, T., and Iino, M. (2007). Identification of the gravitropism-related rice gene LAZY1 and elucidation of LAZY1-dependent and -independent gravity signaling pathways. Plant Cell Physiol. 48, 678-688. doi: 10.1093/pcp/pcm042

Yoshihara, T., and Spalding, E. P. (2017). LAZY Genes Mediate the Effects of Gravity on Auxin Gradients and Plant Architecture. Plant Physiol. 175, 959969. doi: $10.1104 / \mathrm{pp} .17 .00942$
Yoshihara, T., and Spalding, E. P. (2020). Switching the Direction of Stem Gravitropism by Altering Two Amino Acids in AtLAZY1. Plant Physiol. 182, 1039-1051. doi: 10.1104/pp.19. 01144

Yoshihara, T., Spalding, E. P., and Iino, M. (2013). AtLAZY1 is a signaling component required for gravitropism of the Arabidopsis thaliana inflorescence. Plant J. 74, 267-279. doi: 10.1111/tpj.12118

Yu, B., Lin, Z., Li, H., Li, X., Li, J., Wang, Y., et al. (2007). TAC1, a major quantitative trait locus controlling tiller angle in rice. Plant J. 52, 891-898. doi: 10.1111/j.1365-313x.2007.03284.x

Conflict of Interest: The authors declare that the research was conducted in the absence of any commercial or financial relationships that could be construed as a potential conflict of interest.

Copyright (c) 2021 Jiao, Du, Chen, Huang and Ge. This is an open-access article distributed under the terms of the Creative Commons Attribution License (CC BY). The use, distribution or reproduction in other forums is permitted, provided the original author(s) and the copyright owner(s) are credited and that the original publication in this journal is cited, in accordance with accepted academic practice. No use, distribution or reproduction is permitted which does not comply with these terms. 